\title{
Manipulation and Immobilization of Nanostructures for In-situ STEM
}

\author{
Alex W. Robertson ${ }^{1}$, B. Layla Mehdi ${ }^{1,2}$, Libor Kovarik ${ }^{3}$, and Nigel D. Browning ${ }^{1,2,4}$ \\ 1. Physical and Computational Science Directorate, PNNL, Richland, WA 99352, USA. \\ 2. Joint Center for Energy Storage Research (JCESR), Pacific Northwest National Laboratory (PNNL), \\ Richland, WA 99352, USA. \\ 3. Environmental Molecular Sciences Laboratory, PNNL, Richland, WA 99352, USA \\ 4. Materials Science and Engineering, University of Washington, Seattle, WA 98195, USA.
}

Recent developments in in-situ TEM liquid cells have permitted research into the fundamental nanoscale processes that occur during nanoparticle nucleation, growth, and interaction [1]. Several challenges yet remain in the development of this technique; including the development of superior low-dose methods, so that the dose-limited resolution may be improved; implementing sample preparation protocols that minimize inclusion of unwanted additives, such that experiments are more consistent and repeatable; and also to measure and account for the additional role of the electron beam in the observed reaction [2, 3]. A further key difficulty to-date has been that of consistently achieving lattice resolutions, which would enable studies of facet dependent reactions, dislocation-enabled strain accommodation, and the role of grain boundaries and crystal twinning in nanoparticle growth. Pioneering works employing graphene as an ultra-thin membrane to reduce beam scatter demonstrate that such resolutions can be achieved provided the right experimental conditions, however scalable and facile implementation of graphene cells has proven highly demanding [4].

It has been demonstrated that lattice resolution may be achieved in STEM imaging on condition that the sample is stably anchored to the top membrane of the liquid cell (Figure 1a) [5]. The combination of the sample remaining immobile, thus minimizing motion induced artefacts, and that the incident beam has a minimal path length through the scattering liquid, grant these resolutions. However, with current methods obtaining such images relies on good fortune, with little control or consistency. To address this we have developed a functionalization method for the SiN membrane surface that promotes nanoparticle adhesion (Figure 1b), allowing for more consistent lattice resolutions with a relatively straightforward technique.

The experiments were performed using a commercially available liquid-cell within an aberration corrected STEM. We demonstrated the viability of the technique with standard Au nanoparticle samples $(4 \mathrm{~nm}$ diameter, CTAB or citrate terminated) [6]. These were then added to a $2.5 \times 10^{-4} \mathrm{M} \mathrm{HAuCl}_{4}$ growth solution with $0.1 \mathrm{M}$ cetyltrimethylammonium bromide (CTAB), and examined by in-situ STEM. The reducing action of the electron beam was employed to promote the growth of the Au seeds in to larger nanostructures. The action of various additives, such as CTAB concentration, $\mathrm{AgNO}_{3}$, and iodide were studied to determine their role in facet passivation/activation, permitted via lattice resolution imaging, and thus manipulate the preferential growth of particular nanostructure geometries [7].

\section{References:}

[1] F. M. Ross, Science 350, aaa9886 (2015). DOI: 10.1126/science.aaa9886

[2] L. Kovarik et al, Appl. Phys. Lett. 109, 164102 (2016).

[3] J. Kim et al, ACS Nano 10, 9801-9808 (2016).

[4] J. Yuk et al, Science 336, 61-64 (2012). 
[5] T. J. Woehl et al, Ultramicroscopy 127, 53-63 (2013).

[6] N. R. Jana et al, J. Phys. Chem. B 105, 4065-4067 (2001).

[7] The development of the operando stage was supported by the Chemical Imaging Initiative (CII), a Laboratory Directed Research and Development (LDRD) Program at Pacific Northwest National Laboratory (PNNL). The image analytics used for quantification were developed as part of the Analytics In Motion (AIM) Initiative LDRD Program at PNNL. PNNL is a multi-program national laboratory operated by Battelle for the U.S. Department of Energy (DOE) under Contract DE-AC05-76RL01830. A portion of the research was performed using the Environmental Molecular Sciences Laboratory (EMSL), a national scientific user facility sponsored by the Department of Energy's Office of Biological and Environmental Research and located at PNNL.
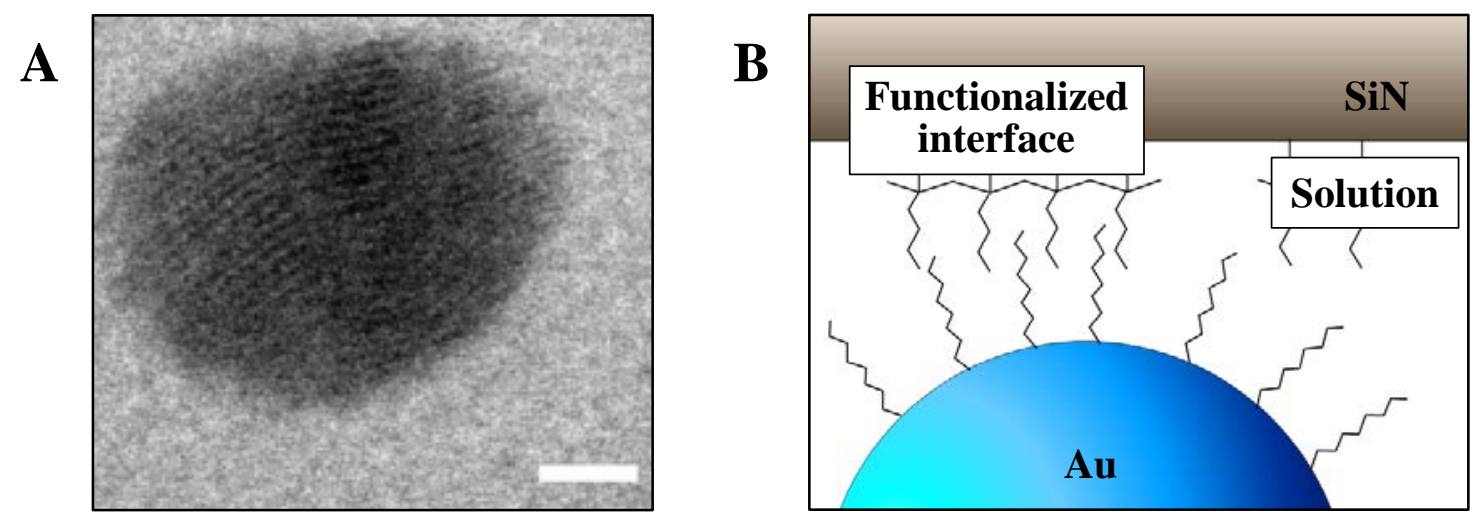

Figure 1. (A) Lattice resolution BF-STEM image of a $\mathrm{PbO}_{2}$ nanoparticle in a liquid cell. Scale bar $2 \mathrm{~nm}$. Reproduced from [5]. (B) Cartoon illustration showing a functionalized SiN membrane on the top of a SiN cell. A ligand terminated Au nanoparticle interacts with the functional groups, adhering to the SiN. 